\title{
Predictors of Adverse Outcomes in Uncomplicated Lower Respiratory Tract Infections
}

\author{
Michael Moore, BMBS, FRCGP' \\ Beth Stuart, $P b D^{1}$ \\ Mark Lown, MBBS, $P b D^{1}$ \\ Ann Van den Bruel, MD, PbD ${ }^{2}$ \\ Sue Smith, $P b D^{2}$ \\ Kyle Knox, MBCbB, MRCGP, \\ $M R C P^{2}$ \\ Matthew J. Thompson, MBCbB, \\ $M P H, D P b i l, M R C G P^{3}$ \\ Paul Little, MBBS, FRCGP \\ 'University of Southampton, Primary Care \\ and Population Sciences, Aldermoor Health \\ Centre, Southampton, United Kingdom \\ ${ }^{2}$ University of Oxford, Nuffield Depart- \\ ment of Primary Health Care Sciences, \\ Radcliffe Observatory Quarter, Oxford, \\ United Kingdom \\ ${ }^{3}$ University of Washington, Seattle, \\ Washington

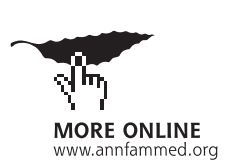

AC Annals Journal Club selection; see inside back cover or http://www. annfammed.org/site/AJC/.

Conflicts of interest: authors report none.

\section{CORRESPONDING AUTHOR}

Michael Moore

University of Southampton

Primary Care and Population Sciences

Aldermoor Health Centre

Southampton SO16 5ST, UK

mvm198@soton.ac.uk

\begin{abstract}
PURPOSE Presentation with acute lower respiratory tract infection (LRTI) in primary care is common. The aim of this study was to help clinicians treat patients presenting with LRTI in primary care by identifying those at risk of serious adverse outcomes (death, admission, late-onset pneumonia).
\end{abstract}

METHODS In a prospective cohort study of patients presenting with LRTI symptoms, patient characteristics and clinical findings were recorded and adverse events identified over 30 days by chart review. Multivariable logistic regression analyses identified predictors of adverse outcomes.

RESULTS Participants were recruited from 522 UK practices in 2009-2013. The analysis was restricted to the 28,846 adult patients not referred immediately to the hospital. Serious adverse outcomes occurred in 325/28,846 (1.1\%). Eight factors were independently predictive; these characterized symptom severity (absence of coryza, fever, chest pain, and clinician-assessed severity), patient vulnerability (age $>65$ years, comorbidity), and physiological impact (oxygen saturation $<95 \%$, low blood pressure). In aggregate, the 8 features had moderate predictive value (area under the receiver operating characteristic curve 0.71, $95 \% \mathrm{Cl}, 0.68-0.74)$; the $4 \%$ of patients with $\geq 5$ features had an approximately 1 in $17(5.7 \%)$ risk of serious adverse outcomes, the $35 \%$ with 3 or 4 features had an intermediate risk (1 in 50, 2.0\%), and the $61 \%$ with $\leq 2$ features had a low (1 in 200, 0.5\%) risk.

CONCLUSIONS In routine practice most patients presenting with LRTI in primary care can be identified as at intermediate or low risk of serious outcome.

Ann Fam Med 2019;17:231-238. https://doi.org/10.1370/afm.2386.

\section{INTRODUCTION}

cute uncomplicated respiratory tract infections are one of the most
common acute illnesses managed in primary care, and the majority
are treated with antibiotics. ${ }^{1-3}$ The Cochrane review of antibiotics for bronchitis reported only small symptomatic benefit from antibiotics, ${ }^{4}$ findings confirmed in the largest clinical trial to date. ${ }^{5}$ Despite the limited effect on symptoms, patients and clinicians are concerned about more severe or prolonged illness and complications. ${ }^{6}$ To aid decision making in the consultation, it would help primary care clinicians if they understood who was at greatest risk of future serious adverse outcomes. Such adverse outcomes are uncommon, and large numbers of subjects are needed to produce robust risk estimates. We report the findings from a large prospective cohort of patients presenting with acute lower respiratory infection (LRTI) in UK primary care who were followed up for 30 days by clinical record review. We have already reported the clinical features that aid diagnosis of pneumonia at first consultation. ${ }^{7}$ This article reports the clinical features that predict future serious adverse outcomes: death, future hospitalization, and late-onset pneumonia (diagnosed $>7$ days after presentation).

The objective of this study was to help clinicians treat patients presenting with LRTI in primary care by identifying those at risk of serious adverse outcomes (death, hospital admission, late-onset pneumonia). 


\section{METHOD}

\section{Key Design Features}

This was a prospective cohort more fully reported elsewhere. ${ }^{8}$ Clinical presenting features and management strategies were documented via structured clinical pro forma at an index consultation. Review of medical records was performed to ascertain x-ray findings, subsequent reconsultations with new or worsening illness, and hospitalization or death during the next 30 days.

\section{Participants}

A cohort of 28,883 adult patients with acute cough attributed to LRTI was recruited from 522 practices in $2009-2013 ; 28,846$ patients not immediately referred to the hospital were eligible for this analysis.

\section{Inclusion Criteria}

Patients had to be aged $\geq 16$ years and presenting with a new illness. We used a pragmatic definition of LRTI consistent with the Cochrane review of antibiotics for bronchitis ${ }^{4}$ : acute cough (new or worsening cough for $\leq 3$ weeks) presenting as the main symptom and judged to be infective in origin by the physician.

\section{Exclusion Criteria}

Patients were excluded if they had other cause of acute cough (eg, heart failure, acid reflux, fibrosing alveolitis), were unable to fill out the diary (eg, severe mental illness, dementia, or mental impairment), were immune compromised, or previously presented with the same episode of illness. These criteria are comparable to those applied in several previous LRTI trials and cohort studies. . $^{5-911}$

\section{Patient Involvement}

Working general practitioners were involved in designing the clinical record form (CRF) and interpreting findings. Patients were involved in preparing the program grant application; advising about the patient information leaflets, consent forms, CRF, and outcomes $_{\text {; }}$ and participating regularly in study management meetings.

\section{Data Collection}

\section{Clinical Record Form}

A clinical data collection form was completed at the point of recruitment by the physician, collecting data on age, smoking history, previous duration of symptoms, nature and severity of symptoms (dry cough, productive cough, shortness of breath, coryza, fever, chills or shivering, chest pain, headache, muscles aches, sleep disturbance, confusion, diarrhea, sputum color), examination (respiratory rate, pulse, blood pressure, oxygen saturation, temperature, presence of wheeze, crepita- tions, or bronchial breathing), a rating of the overall severity of the illness (Visual Analogue Score ranging from "well" to "very unwell"), and whether antibiotics were prescribed. No training was provided to calibrate the severity scale, which reflected the physician's overall assessment of the patient or "gut feeling."

\section{Notes Review}

Data on $\mathrm{x}$-ray findings were collected at notes review. All reports were considered by the authors and rated as definite pneumonia, probable pneumonia, possible pneumonia, unlikely pneumonia, and not pneumonia based on the text in the report. Other diagnoses (ie, tuberculosis and cancer) were also noted, and differences were resolved by discussion to achieve consensus. Outcome data were abstracted by practice staff overseen by either local research network staff or research staff from the Oxford center. Where a clinical diagnosis of pneumonia was recorded in the record without $\mathrm{x}$-ray confirmation, this was accepted as accurate and included in the outcome measure. No further corroboration was possible. The national deprivation index of the place of residence was derived from the patient's postcode. Data submitted by practices on paper forms were double entered by the data management team in Oxford, who also followed up data inconsistencies or missing data with individual practices. We have previously shown that clinical records can be assessed reliably by using a very similar structured pro forma. ${ }^{12}$ Admissions unrelated to the index consultation (eg, elective admissions) were recorded but excluded from the analysis.

\section{Other Data}

Cardiovascular or cerebrovascular morbidities and lung comorbidities noted in the medical records were also documented. Lung comorbidity included acute and chronic obstructive airway disease (ie, asthma, chronic obstructive pulmonary disease) or history of other significant lung disease necessitating hospital investigation and the use of steroids or bronchodilators. Vaccination status (Pneumovax) was also recorded.

\section{Sample Size}

The overall recruitment target of 28,000 patients was originally designed to achieve $80 \%$ power to identify predictive variables of adverse outcomes after LRTI, with an odds ratio of $3(\alpha=0.01)$, on the assumption of an antibiotic prescribing rate of $50 \%$ and an event rate of 0.005 .

\section{Primary Outcome}

Participants were included as cases if there was evidence from the record of death, hospital admission, or 
clinical diagnosis of pneumonia after day 1 (ie, arising 2 to 30 days from presentation). Patients admitted on the day of the consultation were excluded from the analysis because we were interested in predicting subsequent admission or death. On the same basis, we excluded pneumonia diagnosed on the basis of an x-ray report (but without additional consultation) within the first 7 days (assuming this diagnosis was based on $x$-ray investigations requested at the time of the index consultation). Diagnosis of pneumonia was based on a clinical record entry or x-ray report.

\section{Statistical Analysis}

Prediction of Imparted Risk of Adverse Outcome

The explanatory variables assessed were patient characteristics (age, sex, social deprivation, and medical history), presenting symptoms, and clinical signs elicited by examination at the index consultation. Symptoms were included if reported as present, irrespective of their severity. We assessed whether these individual variables were predictors of the outcome in a univariable analysis. Variables that were significantly associated with the outcome $(P<.05)$ were then included in a multivariable model via a generalized linear model for the binomial family with robust clustered variance estimators to allow clustering of patients by doctor. Participants were included regardless of whether they were prescribed antibiotics, and the antibiotic prescription was included in the multivariable model.

\section{Statistical Modeling of Prognostic Values}

The value of combining statistically predictive variables from the multivariable model was assessed by including them in a logistic regression model, starting with the most predictive and sequentially adding in the variables that most increased the area under the receiver operating characteristic curve (AUROC), with 1,000 bootstrapped samples to avoid overfitting. Goodness of fit was assessed by the Hosmer-Lemeshow test. Oxygen saturation is regarded as normal within the range of $95 \%$ to $99 \%$, and so values were dichotomized at $<95 \%$. Temperature was dichotomized at $>37.8^{\circ} \mathrm{C}$ and tachycardia at $>100 \mathrm{bpm}$ to be consistent with previous diagnostic models. ${ }^{13}$ Age and blood pressure cutoffs were chosen to align with the CRB-65 score. ${ }^{14}$

\section{Sensitivity Analyses}

Sensitivity analyses were carried out to assess the effect of varying 4 analytic parameters: (1) definition of pneumonia (by excluding "possible" pneumonia), (2) definition of pneumonia (by excluding cases of pneumonia recorded only in the physician's clinical record and without x-ray confirmation), (3) severity of symptoms (by including symptoms only if reported as severe), and (4) imputation of missing values for $\mathrm{O}_{2}$ saturation (by assuming the extreme positions that all missing values were $<95 \%$ or all were $>95 \%$ ). We did not impute missing data for every variable because levels of missingness were mostly low. ${ }^{7}$

\section{RESULTS}

\section{Frequency of Serious Adverse Outcomes}

Of the cohort of 28,883 participants, 1,782 had a chest $\mathrm{x}$-ray within 30 days, and 1,062 had a chest $\mathrm{x}$-ray between 8 and 30 days. Those referred for chest $\mathrm{x}$-ray were older, more likely to smoke, more severely ill by global assessment, and more likely to have positive physical signs than the whole cohort. The baseline characteristics of the cohort have been reported elsewhere, and the baseline table is reproduced in Supplemental Table 1 (http://www.annfammed.org/ content/17/3/21/supp1/DC1). ${ }^{7}$ Table 1 shows the $\mathrm{x}$-ray results: 120 cases of pneumonia were included in the primary analysis on this basis (ie, only cases assessed as "unlikely" or "not" pneumonia were excluded, pneumonia secondary to cancer or tuberculosis was included). An additional 34 non-x-ray confirmed late-onset "pneumonia" cases were also included in the analysis based on clinical diagnoses recorded in the medical record, and of these 12 were also confirmed by subsequent $\mathrm{x}$-ray or admission.

Thirty-seven of the 28,883 participants were hospitalized on the day of consultation. Among the remaining 28,846, there were 221 hospitalizations and 30 deaths (some deaths occurring after admission). Twenty-five of the hospital admissions and 1 death were unrelated to the index consultation. Respiratory infections accounted for the greatest number of hospitalizations and deaths (respectively 132 and 7); there were 20 hospitalizations and 8 deaths from cardiovascular and cerebrovascular events, 20 hospitalizations and 4 deaths from other circulatory problems (dehydration, renal failure, "collapse"), 12 hospitalizations and 9 deaths from cancer, and 12 hospitalizations and

Table 1. Attribution of Diagnosis Reported on X-rays for All Reports and Those 8 to 30 Days

\begin{tabular}{lcc}
\hline & All X-rays & X-rays 8-30 Days \\
\hline Not pneumonia & $1,539(86.4 \%)$ & $938(88.3 \%)$ \\
Unlikely pneumonia & $8(0.5 \%)$ & $4(0.4 \%)$ \\
Definitely pneumonia & $184(10.3 \%)$ & $95(9.0 \%)$ \\
Probable pneumonia & $28(1.6 \%)$ & $12(1.1 \%)$ \\
Possible pneumonia & $18(1.0 \%)$ & $9(0.9 \%)$ \\
Cancer & $4(0.2 \%)$ & $3(0.3 \%)$ \\
Tuberculosis & $1(0.1 \%)$ & $1(0.1 \%)$ \\
\hline
\end{tabular}


1 death from other infections. Late-onset pneumonia was present in 34 patients, with subsequent $x$-ray confirmation and admission in 12 .

In summary, the frequency of $\geq 1$ serious adverse events potentially related to the initial consultation for LRTI (and therefore included in the predictive analysis) was $1.1 \%(325 / 28,846)$, with 29 deaths $(0.1 \%)$, 120 cases of late-onset pneumonia (including cancer or tuberculosis) (0.4\%), and 196 hospital admissions occurring after the date of the index consultation $(0.7 \%)$ (Table 2$)$.

\section{Predictors of Severe Adverse Outcomes}

Table 3 shows the prognostic value for severe adverse outcomes, expressed as adjusted risk ratios, of the patient characteristics, presenting symptoms, and clinical examination findings at the initial consultation for LRTI. There was evidence of clustering of the outcome at the physician level, with an intraclass correlation coefficient of 0.06 (95\% CI, 0.03-0.13) and therefore the adjusted model uses robust clustered variance estimates.

\section{Developing a Clinical Prediction Score}

Taking forward the variables that are statistically significant within each group and entering them into a multivariable regression model, starting with the most predictive, reveals 8 independent predictors of serious adverse outcomes (at the 1\% probability level). These independent predictors are oxygen saturation $<95 \%$, age $\geq 65$ years, low blood pressure, fever, comorbidity, no coryza, severity score $>5 / 10$, and chest pain (Table 4).

A simple score based on the presence or absence of each of these 8 items where each is assigned a value of 1, based on 1,000 bootstrapped samples, has an AUROC of 0.71 (95\% CI, 0.68-0.74). Table 5 shows how such a score is likely to be distributed in the population (percentages are based on the number of participants in the cohort with complete data on these 8 items). Using continuous variables for oxygen saturation, age, blood pressure, temperature, and sever- ity score, the AUROC is 0.74 (95\% CI, 0.71-0.77). Although using continuous variables would improve predictive values, the confidence interval overlaps with the score using binary presence and absence variables, which is easier to translate into clinical practice.

\section{Sensitivity Analyses}

Clinicians traditionally give more weight to lateralizing (asymmetric) symptoms. Treating wheezing, crackles, and bronchial breathing as categorical (none, unilateral, or bilateral) does not add precision; although significant in the univariable analysis, they are not significant in the multivariable analysis and are not included in the final model. Excluding the "possible pneumonia" from the model reduced the number of pneumonia cases to 106 but did not change the predictive variables selected. Similarly, excluding all but severe symptoms from the analysis had little impact: chest pain was excluded, but severe shortness of breath and severe chills were included. The AUROC for this model was also similar: 0.70 (95\% CI, 0.67 $0.73)$. Excluding cases of late-onset pneumonia only recorded in the clinical record and without subsequent admission or $\mathrm{x}$-ray did not change the variables selected, AUROC 0.71 (95\% CI, 0.68-0.74).

Imputing missing values for oxygen saturation had little impact on the assessed relative risk or on the statistical model and did not improve the model's discrimination. Assuming all missing oxygen saturation values were $<95 \%$ gave an AUROC of 0.69 (95\% CI, $0.66-0.72)$, and assuming all the missing values were $>95 \%$ gave an AUROC of 0.68 (95\% CI, 0.66-0.71). The Hosmer-Lemeshow test indicated that calibration is poor $(P<.001)$ for all models.

\section{Diagnostic Performance of an 8-Item Score in Clinical Practice}

Table 6 shows the probable prognostic performance of the predictive variables in clinical practice. Using the score would enable the clinician to identify high-, intermediate-, and low-risk groups.

Table 2. Contribution of Categories to Total Adverse Event Tally

\begin{tabular}{|c|c|c|c|c|c|c|}
\hline & \multirow[b]{2}{*}{ Total } & \multicolumn{4}{|c|}{ Also Contributed to This Category } & \multirow{2}{*}{$\begin{array}{c}\text { Total Excluding } \\
\text { Duplicates (Hierarchy: } \\
\text { Death, Admission, } \\
\text { Pneumonia) }\end{array}$} \\
\hline & & $\begin{array}{l}\text { X-ray Pneumonia } \\
>1 \mathrm{wk}\end{array}$ & $\begin{array}{c}\text { Clinical (Notes Review) } \\
\text { Pneumonia }\end{array}$ & $\begin{array}{l}\text { Admission } \\
\text { After Day } 1\end{array}$ & Death & \\
\hline X-ray pneumonia $>1 \mathrm{w}$ & 120 & N/A & 5 & 23 & 0 & 92 \\
\hline $\begin{array}{l}\text { Clinical (notes review) } \\
\text { pneumonia }\end{array}$ & 34 & 5 & N/A & 6 & 1 & 22 \\
\hline Admission after day 1 & 196 & 23 & 6 & N/A & 14 & 182 \\
\hline \multirow[t]{2}{*}{ Death } & 29 & 0 & 1 & 14 & N/A & 29 \\
\hline & & & & & & Total: 325 \\
\hline
\end{tabular}




\section{DISCUSSION}

\section{Principal Findings}

Serious adverse outcomes (late-onset pneumonia, admission, death) are uncommon after presentation with uncomplicated LRTI $(1.1 \%, 325 / 28,846)$, and in almost one-half of cases of hospital admission and death $(44.4 \%$ of all cases, $76 \%$ of deaths) respiratory infection was not stated as the primary cause on the discharge summary or death certificate. The likelihood of a serious adverse outcome depends on 3 factors: symptom severity (absence of coryza, fever, chest pain, and clinician-assessed severity), patient vulnerability (age $\geq 65$ years, comorbidity), and physiological impact (oxygen saturation $<95 \%$, low blood pressure). These 8 individual features can be used to predict adverse outcomes by conversion to an 8 -point score.

The reported causes of hospitalization and death in this study highlight that disease progression to a serious outcome is not simply a case of worsening respiratory infection, so it is perhaps not surprising that previous analysis of this cohort did not convincingly demonstrate that prescribing of antibiotics reduces the risk of death or admission. ${ }^{8}$ Cardiovascular morbidity is common, and antibiotics may not be the most

Table 3. Risk Factors at First Consultation for Severe Adverse Outcomes (Death or Hospitalization From Lower Respiratory Tract Infection Complications Within 30 Days or Late Onset or Pneumonia Confirmed by X-ray or Reconsultation 8 to 30 Days After First Consultation) $(n=325)$

\begin{tabular}{|c|c|c|c|c|c|c|}
\hline & \multicolumn{2}{|c|}{$\begin{array}{c}\text { Proportion of Patients Suffering } \\
\text { Adverse Outcome }\end{array}$} & \multicolumn{2}{|c|}{ Risk Ratio } & \multicolumn{2}{|c|}{ Adjusted Risk Ratio } \\
\hline & $\begin{array}{l}\text { Characteristic + } \\
\text { No. (\%) }\end{array}$ & $\begin{array}{l}\text { Characteristic - } \\
\text { No. (\%) }\end{array}$ & Ratio $(95 \% \mathrm{Cl})$ & $P$ Value & Ratio $(95 \% \mathrm{Cl})$ & $P$ Value \\
\hline \multicolumn{7}{|l|}{ Patient characteristics } \\
\hline Age $\geq 65$ years & $163 / 7,921(2.1)$ & $162 / 20,925(0.8)$ & $2.66(2.14-3.30)$ & $<0.001$ & $2.15(1.72-2.67)$ & $<0.001$ \\
\hline Male & $157 / 11,743(1.3)$ & $16 / 17,098$ (0.09) & $1.36(1.10-1.70)$ & 0.005 & $1.20(0.96-1.50)$ & 0.112 \\
\hline Influenza vaccine & $152 / 9,842(1.5)$ & $173 / 19,004(0.9)$ & $170(1.37-2.11)$ & $<0.001$ & $0.80(0.61-1.05)$ & 0.110 \\
\hline Pneumovax $<10$ y & $95 / 5,294(1.8)$ & $230 / 23,552(1.0)$ & $1.84(1.45-2.33)$ & $<0.001$ & $1.01(0.79-1.29)$ & 0.939 \\
\hline Ever smoked & $1,997 / 15,165(13.1)$ & $120 / 13,212(0.9)$ & $1.43(1.14-1.79)$ & 0.002 & $1.25(0.99-1.57)$ & 0.060 \\
\hline Any comorbidity & $210 / 13,100(1.6)$ & $115 / 15,746(0.7)$ & $2.19(1.75-2.75)$ & $<0.001$ & 1.57 (1.24-1.99) & $<0.001$ \\
\hline Lung comorbidity & $112 / 7,461(1.5)$ & $213 / 21,385(1.0)$ & $1.51(1.20-1.89)$ & $<0.001$ & $1.00(0.75-1.32)$ & 0.976 \\
\hline Steroids or bronchodilators & $93 / 6,537(1.4)$ & $218 / 20,997(1.0)$ & $1.37(1.08-1.75)$ & 0.010 & $0.87(0.67-1.12)$ & 0.278 \\
\hline Living in deprived area ${ }^{b}$ & $69 / 5,750(1.2)$ & $256 / 23,096(1.1)$ & $1.08(0.83-1.41)$ & 0.556 & $1.10(0.81-1.50)$ & 0.530 \\
\hline \multicolumn{7}{|l|}{ Presenting symptoms } \\
\hline Shortness of breath & $246 / 18,498(1.3)$ & $77 / 10,229(0.8)$ & $1.77(1.37-2.28)$ & $<0.001$ & $1.55(1.18-2.04)$ & 0.002 \\
\hline Fever & $126 / 10,978(1.1)$ & $198 / 17,800(1.1)$ & $1.03(0.83-1.29)$ & 0.782 & $1.01(0.80-1.29)$ & 0.919 \\
\hline Chills & $128 / 9,146(1.4)$ & $195 / 19,621(1.0)$ & $1.41(1.13-1.76)$ & 0.002 & $1.41(1.11-1.79)$ & 0.005 \\
\hline Chest pain & $146 / 10,644(1.4)$ & $178 / 18,130(1.0)$ & $1.40(1.12-1.74)$ & 0.003 & $1.31(1.03-1.67)$ & 0.028 \\
\hline Confusion & $25 / 1,860(1.3)$ & $300 / 26,968(1.1)$ & $1.21(0.81-1.81)$ & 0.360 & $1.11(0.75-1.65)$ & 0.605 \\
\hline No coryza & $184 / 13,029(1.4)$ & $139 / 15,718(0.9)$ & $1.60(1.28-1.99)$ & $<0.001$ & $1.60(1.27-2.02)$ & $<0.001$ \\
\hline Headache & $131 / 13,254(1.0)$ & $193 / 15,507(1.2)$ & $0.79(0.64-0.99)$ & 0.041 & $0.73(0.57-0.94)$ & 0.014 \\
\hline Muscle aches & $112 / 10,497(1.1)$ & $211 / 18,267(1.2)$ & $0.92(0.74-1.16)$ & 0.495 & $0.82(0.63-1.07)$ & 0.140 \\
\hline Diarrhea & $27 / 2,508(1.1)$ & $298 / 26,312(1.1)$ & $0.95(0.64-1.41)$ & 0.800 & $0.89(0.60-1.31)$ & 0.557 \\
\hline Sputum: purulent & $186 / 18,221(1.0)$ & $139 / 10,621(1.3)$ & $0.78(0.63-0.97)$ & 0.026 & $0.70(0.57-0.87)$ & 0.001 \\
\hline Sputum bloody or rusty & $16 / 1,023(1.6)$ & $309 / 27,819(1.1)$ & $1.19(0.92-1.52)$ & 0.179 & $0.99(0.76-1.27)$ & 0.917 \\
\hline \multicolumn{7}{|l|}{ Clinical examination findings } \\
\hline Severity assessment >5/10 & $198 / 11,893(1.7)$ & $126 / 16,943(0.7)$ & $2.24(1.79-2.80)$ & $<0.001$ & $1.48(1.13-1.93)$ & 0.004 \\
\hline Respiratory rate $>24 / \mathrm{min}$ & $61 / 2,885(2.1)$ & $263 / 25,844(1.0)$ & $2.08(1.58-2.74)$ & $<0.001$ & $1.42(1.07-1.88)$ & 0.016 \\
\hline Temperature $>37.8^{\circ} \mathrm{C}$ & $40 / 1,656(2.4)$ & $28 / 27,169(0.1)$ & $2.31(1.67-3.21)$ & $<0.001$ & $1.82(1.28-2.58)$ & 0.001 \\
\hline Pulse $>100 / \mathrm{min}$ & $45 / 2,801(1.6)$ & $280 / 26,033(1.1)$ & $1.49(1.09-2.04)$ & 0.012 & $1.03(0.75-1.40)$ & 0.858 \\
\hline $\mathrm{O}_{2}$ sat $<95 \%$ & $60 / 1,698(3.5)$ & 205/22,047 (0.9) & $3.80(2.86-5.05)$ & $<0.001$ & $2.76(2.08-3.65)$ & $<0.001$ \\
\hline $\mathrm{SBP}<90$ or $\mathrm{DBP}<60 \mathrm{~mm} \mathrm{Hg}$ & $39 / 2,193(1.8)$ & $286 / 26,653(1.1)$ & $1.66(1.19-2.31)$ & 0.003 & $1.72(1.20-2.46)$ & 0.003 \\
\hline Crackles & $1,922 / 12,256(15.7)$ & $133 / 16,582(0.8)$ & $1.95(1.57-2.43)$ & $<0.001$ & $1.39(1.01-1.90)$ & 0.044 \\
\hline Bronchial breathing & $37 / 2,166(1.7)$ & $288 / 26,667(1.1)$ & $1.58(1.13-2.22)$ & 0.008 & $1.07(0.71-1.60)$ & 0.758 \\
\hline Wheezing & $107 / 7,071(1.5)$ & $218 / 21,765(1.0)$ & $1.51(1.20-1.90)$ & $<0.001$ & $0.89(0.67-1.18)$ & 0.412 \\
\hline
\end{tabular}


Table 4. Independent Predictors of Adverse Outcome $(P<.01)$

\begin{tabular}{lcc}
\hline & $\begin{array}{c}\text { Risk Ratio } \\
(\mathbf{9 5 \%} \mathbf{C l})\end{array}$ & $\boldsymbol{P}$ Value \\
\hline $\mathrm{O}_{2}$ sat $<95 \%$ & $2.30(1.74-3.04)$ & $<.001$ \\
Age $\geq 65$ years & $2.3(1.65-2.75)$ & $<.001$ \\
$\mathrm{SBP}<90$ or DBP $<60 \mathrm{~mm} \mathrm{Hg}$ & $1.59(1.13-2.25)$ & 0.008 \\
Temperature $>37.8^{\circ} \mathrm{C}$ & $1.81(1.32-2.47)$ & $<.001$ \\
Any comorbidity & $1.55(1.17-2.05)$ & 0.002 \\
No coryza & $1.50(1.17-1.92)$ & 0.001 \\
Severity assessment $>5 / 10$ & $1.45(1.11-1.90)$ & 0.007 \\
Chest pain & $1.43(1.11-1 \cdot 86)$ & 0.006 \\
\hline SBP $=$ systolic blood pressure; DBP = diastolic blood pressure. & \\
aAny comorbidity as defined in the notes review. & \\
\hline
\end{tabular}

important treatment to prevent progression; moreover, antibiotic-induced vomiting and diarrhea may precipitate dehydration, another potential contributor to the nonrespiratory admissions.

\section{Strengths and Limitations}

The main strengths of the study are as follows: (1) The statistical power of the study was high because of the substantial size of the cohort (>28,000 participants); (2) the completeness of follow-up via notes review was high; $_{i}$ (3) the study included patients from routine consultations and was designed for very easy recruitment, to create little or no selection bias and a large generalizable cohort; (4) those recruiting for the study represented a wide range of practices and doctors d $_{i}(5)$ the diagnosis of chest infections was based on criteria similar to those used in the Cochrane review ${ }^{4}$ and in other studies in primary care ${ }^{5,9-11}$; and

(6) the clinical characteristics of included participants were similar to previous observational cohorts and trials in primary care. 5,9-11,17

An important limitation was the absence of previous training or standardization of recorded history or clinical features (although this increases the generalizability of our findings). There may be incomplete recording of consultation details in routine records, although this limitation is unlikely to pertain to details included in the primary outcomes of interest. Patients were also recruited at the busiest times of year, and, as with

\section{Table 5. Distribution of the Score in the} Presenting Population

\begin{tabular}{lc}
\hline Score & $\mathbf{N}(\%)$ of \\
\hline None & $1,982(8.4)$ \\
1 & $5,529(23.4)$ \\
2 & $6,946(29.4)$ \\
3 & $5,482(23.2)$ \\
4 & $2,672(11.3)$ \\
5 & $783(3.3)$ \\
6 & $201(0.9$ \\
7 & $18(0.1)$ \\
8 & $3(0.01)$ \\
\hline The denominator is 23,616, representing participants with complete data for \\
all variables in the model.
\end{tabular}

other studies of acute infection, ${ }_{1}^{18,19}$ documentation of the details of patients not approached was poor because of time pressures.

The analysis does not take into account the risk of pneumonia diagnosed by $\mathrm{x}$-ray without an additional consultation in the first 7 days, because these were assumed to be related to requests made at the index consultation. The inclusion in the model of $x$-ray diagnosis of pneumonia only after 7 days relates to the potential delayed reporting of routine x-rays in UK practice and may not apply in other settings. We have reported the clinical signs and symptoms associated with early diagnosis of pneumonia elsewhere ${ }^{7}$ t the focus of this model is on late complications, diagnosis, and admission.

Although approximately $20 \%$ of patients had missing data for oxygen saturation, the sensitivity analyses

\begin{tabular}{|c|c|c|c|c|c|c|c|}
\hline $\begin{array}{l}\text { Cutoff } \\
\text { Score } \\
\text { to Use }\end{array}$ & $\begin{array}{l}\text { N (\%) of } \\
\text { Total Cohort }\end{array}$ & $\begin{array}{c}\text { Sensitivity, } \\
\%\end{array}$ & $\begin{array}{c}\text { Specificity, } \\
\%\end{array}$ & $\begin{array}{l}\text { NPV, } \\
\%\end{array}$ & $\begin{array}{l}\text { PPV, } \\
\%\end{array}$ & LR+ & LR- \\
\hline$\geq 1$ & 21,634 (91.6) & 99.2 & 8.5 & 99.9 & 1.2 & 1.08 & 0.09 \\
\hline$\geq 2$ & $16,105(68.2)$ & 91.6 & 32.1 & 99.7 & 1.5 & 1.35 & 0.26 \\
\hline$\geq 3$ & $9,159(38.8)$ & 69.8 & 61.6 & 99.5 & 2.0 & 1.82 & 0.49 \\
\hline$\geq 4$ & $3,677(15.6)$ & 39.3 & 84.7 & 99.2 & 2.8 & 2.57 & 0.72 \\
\hline$\geq 5$ & $1,005(4.3)$ & 21.8 & 95.9 & 99.1 & 5.7 & 5.36 & 0.82 \\
\hline$\geq 6$ & $222(0.9)$ & 8.4 & 99.1 & 99.0 & 9.9 & 9.81 & 0.92 \\
\hline$\geq 7$ & $21(0.1)$ & 1.9 & 99.9 & 98.9 & 23.8 & 27.86 & 0.98 \\
\hline$\geq 8$ & $3(0.01)$ & 0.8 & 100.0 & 98.9 & 66.7 & 178.27 & 0.99 \\
\hline$\leq 2$ & $14,457(61.2)$ & 30.2 & 38.4 & 98.0 & 0.5 & 0.49 & 1.82 \\
\hline $3-4$ & $8,154(34.5)$ & 69.8 & 61.6 & 99.5 & 2.0 & 1.82 & 0.49 \\
\hline$\geq 5$ & $1,005(4.3)$ & 21.8 & 95.9 & 99.1 & 5.7 & 5.36 & 0.82 \\
\hline
\end{tabular}


that imputed missing values for the model did not alter the inferences. A clinical diagnosis of pneumonia in the record was accepted as accurate, but there is evidence to support physician diagnosis of pneumonia being specific but lacking sensitivity. ${ }^{20} \mathrm{~A}$ sensitivity analysis excluding those with a clinical diagnosis of pneumonia but without $\mathrm{x}$-ray confirmation or admission did not alter the model items or performance. It is likely that in this population, where a small proportion received $\mathrm{x}$-rays, there was underascertainment of pneumonic infiltrates. We are therefore limited in this analysis to those with more severe outcomes (admission, death, $\mathrm{x}$-ray pneumonia, or clinical pneumonia) that came to the attention of the attending physician.

\section{Comparison With the Literature}

We are not aware of any comparable cohort studies powered to determine the frequency and predictors of longer-term adverse outcomes, and even the largest randomized trials are underpowered in this respect. For instance, in the largest randomized trial of LRTI to date there were only 3 admissions in the month after randomization in the 2,061 participants. ${ }^{5}$ The predictors of adverse outcomes in this study differ somewhat from the predictors of pneumonia., ${ }^{7,21,22}$ Fever and absence of coryza are the shared items and point to a more severe index illness, whereas some of the model factors for longer-term adverse outcomes probably reflect to a greater extent the individual susceptibility to complications (age comorbidity).

\section{Clinical Implications}

This is the first study to provide robust estimates of the likely frequency and predictors of longer-term adverse outcomes after presentation with LRTI in primary care. As a minimum, our data show that most patients presenting in this way are not going to suffer serious adverse outcomes.

Our findings are likely to be useful to clinicians and help them target antibiotic use in patients with symptoms of LRTI but not pneumonia on examination. Patients scoring $\leq 2$ on the 8 -point scale are at low risk ( 1 in 200) of adverse outcomes, whereas a score $\geq 5$ defines a group as having a $>5 \%$ risk of serious adverse outcomes.

Understanding the factors predicting a higher risk of adverse outcomes could prompt clinicians to more closely follow up patients thus identified — not simply to identify development of late-onset pneumonia but also to recognize and treat appropriately the other causes of admission or death that we have documented, which may have been triggered by an initial respiratory illness ${ }^{23,24}$ Whether such approaches will modify adverse outcomes is unknown.

\section{CONCLUSIONS}

Serious adverse outcomes (late-onset pneumonia, admission, death) occur in only $1.1 \%$ of patients after presentation with LRTI but may be predicted with moderate accuracy by assessment of symptom severity, patient vulnerability, and physiological impact. Eight individual features can be used clinically by conversion to an 8-point score. It is already clear that patients derive little or no symptomatic benefit from antibiotics, and the use of the score may give clinicians more confidence to target prescribing on the basis of predicted risk while highlighting a much smaller higher-risk group of patients who may benefit from closer monitoring.

To read or post commentaries in response to this article, see it online at http://www.AnnFamMed.org/content/17/3/231.

Key words: respiratory tract infections; primary health care; decision support techniques; prognosis

Submitted July 31, 2018; submitted, revised, January 31, 2019; accepted February 28, 2019.

Funding support: NIHR Programme Grant for Applied Research: RP-PG-0407-10098.

Disclaimers: This article presents independent research funded by the National Institute for Health Research (NIHR) under its Programme Grants for Applied Research Programme (reference number RP-PG-040710098). The views expressed are those of the author(s) and not necessarily those of the NHS, the NIHR, or the Department of Health.

Ethical approval: Oxfordshire REC A 09/HO604/67.

UKCRN Portfolio registration number: 7647.

The manuscript is an honest, accurate, and transparent account of the study being reported; that no important aspects of the study have been omitted; and that any discrepancies from the study as planned have been explained.

Author contributions: M.M. developed the protocol for funding and contributed to the management of the study, developed the detailed plan for analysis, and led the drafting of the article and is the guarantor for the article. S.S. developed the protocol; provided day-to-day overall management of the study; coordinated recruitment, follow-up, and data entry; and commented on drafts of the article. P.L. had the original idea for the protocol, led the funding application, supervised the running of the study, contributed to the analysis, and contributed to the drafting of the article. M.J.T. and S.S. led the development of the protocol for ethical approval, supervised the study, and contributed to the analysis and drafting of the article. K.K. and M.L. helped develop the protocol and contributed to developing the detailed plan for analysis, the analysis, and the drafting of the article. A.V.d.B. helped develop the protocol and contributed to developing the detailed plan for analysis and contributed to the drafting of the article. B.S. developed the analysis protocol and led the quantitative analysis, with M.M. and P.L., and contributed to drafting the article.

The datasets used or analyzed during the current study are available from the corresponding author on reasonable request. 
Supplementary materials: Available at http://www.AnnFamMed. org/content/17/3/231/suppl/DC1/.

\section{References}

1. Gulliford MC, Dregan A, Moore MV, et al. Continued high rates of antibiotic prescribing to adults with respiratory tract infection: survey of 568 UK general practices. BMJ Open. 2014;4(10):e006245.

2. Hawker JI, Smith S, Smith GE, et al. Trends in antibiotic prescribing in primary care for clinical syndromes subject to national recommendations to reduce antibiotic resistance, UK 1995-2011: analysis of a large database of primary care consultations. J Antimicrob Chemother. 2014;69(12):3423-3430.

3. Lee GC, Reveles KR, Attridge RT, et al. Outpatient antibiotic prescribing in the United States: 2000 to 2010. BMC Med. 2014;12:96

4. Smith SM, Fahey T, Smucny J, Becker LA. Antibiotics for acute bronchitis. Cochrane Database Syst Rev. 2014;3(3):CD000245.

5. Little P, Stuart B, Moore M, et al; GRACE Consortium. Amoxicillin for acute lower-respiratory-tract infection in primary care when pneumonia is not suspected: a 12-country, randomised, placebocontrolled trial. Lancet Infect Dis. 2013;13(2):123-129.

6. Cornford CS. Why patients consult when they cough: a comparison of consulting and non-consulting patients. Br J Gen Pract. 1998; 48(436):1751-1754.

7. Moore $M$, Stuart B, Little $P$, et al. Predictors of pneumonia in lower respiratory tract infections: $3 C$ prospective cough complication cohort study. Eur Respir J. 2017;50(5):1700434.

8. Little P, Stuart B, Smith S, et al. Antibiotic prescription strategies and adverse outcome for uncomplicated lower respiratory tract infections: prospective cough complication cohort (3C) study. BMJ. 2017;357:j2148.

9. Macfarlane J, Holmes W, Gard P, et al. Prospective study of the incidence, aetiology and outcome of adult lower respiratory tract illness in the community. Thorax. 2001;56(2):109-114.

10. Little P, Rumsby K, Kelly J, et al. Information leaflet and antibiotic prescribing strategies for acute lower respiratory tract infection: a randomized controlled trial. JAMA. 2005;293(24):3029-3035.

11. Butler CC, Hood K, Verheij $T$, et al. Variation in antibiotic prescribing and its impact on recovery in patients with acute cough in primary care: prospective study in 13 countries. BMJ. 2009;338:b2242.

12. Little P, Stuart B, Hobbs FD, et al; DESCARTE investigators. Antibiotic prescription strategies for acute sore throat: a prospective observational cohort study. Lancet Infect Dis. 2014;14(3):213-219.
13. van Vugt SF, Broekhuizen BD, Lammens C, et al; GRACE Consortium. Use of serum $C$ reactive protein and procalcitonin concentrations in addition to symptoms and signs to predict pneumonia in patients presenting to primary care with acute cough: diagnostic study. BMJ. 2013;346:\{2450.

14. McNally M, Curtain J, O'Brien KK, Dimitrov BD, Fahey T. Validity of British Thoracic Society guidance (the CRB-65 rule) for predicting the severity of pneumonia in general practice: systematic review and meta-analysis. Br J Gen Pract. 2010;60(579):e423-e433.

15. Gulliford MC, Moore MV, Little $P$, et al. Safety of reduced antibiotic prescribing for self limiting respiratory tract infections in primary care: cohort study using electronic health records. BMJ. 2016;354: i3410.

16. Teepe J, Little P, Elshof N, et al; GRACE Consortium. Amoxicillin for clinically unsuspected pneumonia in primary care: subgroup analysis. Eur Respir J. 2016;47(1):327-330.

17. Little P, Stuart B, Francis N, et al; GRACE Consortium. Effects of Internet-based training on antibiotic prescribing rates for acute respiratory-tract infections: a multinational, cluster, randomised, factorial, controlled trial. Lancet. 2013;382(9899):1175-1182.

18. Little P, Gould C, Williamson I, Moore M, Warner G, Dunleavey J. Pragmatic randomised controlled trial of two prescribing strategies for childhood acute otitis media. BMJ. 2001;322(7282):336-342.

19. Little P, Stuart B, Hobbs FD, et al; DESCARTE investigators. Predictors of suppurative complications for acute sore throat in primary care: prospective clinical cohort study. BMJ. 2013;347:f6867.

20. van Vugt SF, Verheij TJ, de Jong PA, et al; GRACE Project Group. Diagnosing pneumonia in patients with acute cough: clinical judgment compared to chest radiography. Eur Respir J. 2013;42(4): 1076-1082.

21. van Vugt SF, Broekhuizen BD, Zuithoff NP, et al; GRACE Consortium. Validity of a clinical model to predict influenza in patients presenting with symptoms of lower respiratory tract infection in primary care. Fam Pract. 2015;32(4):408-414.

22. Schierenberg A, Minnaard MC, Hopstaken RM, et al. External validation of prediction models for pneumonia in primary care patients with lower respiratory tract infection: an individual patient data meta-analysis. PLoS One. 2016;11(2):e0149895.

23. Smeeth $L$, Thomas SL, Hall AJ, Hubbard R, Farrington P, Vallance $P$. Risk of myocardial infarction and stroke after acute infection or vaccination. N Engl J Med. 2004;351(25):2611-2618.

24. Kwong JC, Schwartz KL, Campitelli MA, et al. Acute myocardial infarction after laboratory-confirmed influenza infection. $N$ Engl J Med. 2018;378(4):345-353. 